\title{
Machine Learning for Name Type Classification in Library Metadata
}

Mark Edward Phillips

University of North Texas, USA. mark.phillips@unt.edu

\begin{abstract}
This poster reports on the effectiveness of machine learning approaches to classify common names in library metadata records using the Library of Congress Name Authority File. Features extracted from this dataset were used to train and evaluate classification algorithms including decision tree, naïve Bayes, random forest and support vector machine implemented in Weka, an open-source machine learning platform. The best performing classifiers were also tested on a collection of 30,000 names extracted from the UNT Digital Library This poster presents the feature sets, their testing results and the information gains of extracted features. The study demonstrated that machine learning could effectively classify names as persons or corporations.
\end{abstract}

\section{KEYWORDS}

name type classification, digital library metadata, name classification

\section{INTRODUCTION}

Library metadata describes resources and objects using standards and schemes for encoding and representation. Metadata schemes may use different fields for storing and representing name information, e.g., machine-readable cataloging (MARC) designates "creator" field usage by name type (personal, corporate or meeting) and main or added entries (Library of Congress Network Development and MARC Standards Office, 2016). The Metadata Object Description Schema (MODS) has a repeatable name element with an attribute of "type" to denote personal, corporate or event names (Library of Congress, 2012). The Dublin Core Metadata Element Set has "creator" and "contributor" fields but cannot store types (Dublin Core Metadata Initiative, 2012). Schema.org is a vocabulary used on the web to describe resources shared or embedded online and acts as a standardized vocabulary; it defines two classes for person and organization names (Schema.org, n.d.).

Absence of name types is a known barrier to information retrieval. First, knowing the type can facilitate high precision information retrieval, e.g., to help a user retrieve documents mentioning a given name (Paik, Liddy \& Yu 1996). Second, it can help natural language processing systems disambiguate names. Last, a digital library could organize names in browsable lists by type for users.

$80^{\text {th }}$ Annual Meeting of the Association for Information Science \& Technology, Washington, DC | Oct. 27-Nov. 1, 2017

Authors Retain Copyright

\author{
Jiangping Chen \\ University of North Texas, USA. Jiangping.Chen@unt.edu
}

\section{RELATED WORK}

Literature is sparse regarding name type classification and often pairs named entity recognition (NER) with named entity classification (NEC). NER is the identification of boundaries within a text where named entities occur, and NEC is the task of identifying the type of named entity. Both supervised (Heng \& Grishman, 2006) and unsupervised (Collins \& Singer, 1999) approaches have been used for NER and NEC tasks. Name ethnicity classification, a related area, focuses entirely on classifying personal names by ethnicity or region (Mateos, 2007).

A commercially-available tool called NameSifter (IBM, n.d) "examines a name and attempts to tell you what kind of entity (person, organization, something else) that the name represents."

Type classification on name strings is not easily identified within literature on machine learning, digital libraries or library metadata.

\section{METHODS}

This study investigated the application of machine-learning technologies to the automatic classification of name strings as personal or corporate names, based on three research questions: 1) Can we teach a model to automatically classify names? 2) Which machine learning approach works best? 3) Which features are most useful in classifying names?

\section{Dataset}

This research used the Library of Congress Name Authority File (LC-NAF) from the LC Linked Data Service (Library of Congress, n.d). It is a large $(\mathrm{n}=7,861,721)$ set of public data containing both personal $(6,361,899,81 \%)$ and corporate $(1,499,822,19 \%)$ names and is representative of bibliographic metadata. Long-standing practices and standards dictate the formatting of names, e.g., inverting the order of the surname and given names (Last, First).

\section{Feature Selection}

We identified features of name strings that could help classify them and relevant dictionaries for common names and abbreviations. In total 27 features were tested and used in the building of classification models.

\section{RESULTS}

\section{Performance of ML Classifiers}

We experimented with classifiers implemented in Weka, a machine-learning platform, including decision tree (DT), na- 
ïve Bayes (NB), random forest (RF), and support vector machines (SVM), trained on the identified features. We tuned model hyper-parameters in 10-fold cross-validation experiments (Train + ) and tested the models against 10,000 names in a test set (Test) from the dataset. A total of 31 classifiers were compared in this research. The best performing classifiers were also tested against a set of 30,000 names extracted from the UNT Digital Library (https://digital.library.unt.edu) as an external "real world" test dataset.

The three best performing classifiers using the selected features were the J48 decision tree classifier (an open source Java implementation of the C4.5 algorithm), random forest (RF) and a logistic model tree (LMT) classifier (see Table 1).

\section{DISCUSSION AND CONCLUSION}

\section{Significance of the Study}

This study was the first exploring the LC-NAF dataset for automatic name type classification on real-world metadata values. Our study showed that it was possible to teach highperformance models to classify name strings, and that some features (e.g., commas and comma placement) play an important role.

\section{Conclusion}

We addressed the classification of names as personal or corporate by experimenting with 27 features and training 31 classifiers and showed that it is possible to automatically classify library name strings as personal and corporate names. Our study identified three classifiers with high precision, recall, accuracy, f1-measure and ROC area. Future work will explore machine learn ing to identify and classify named entities in library records spanning multiple elements.

\begin{tabular}{|l|l|l|l|l|l|}
\hline Classifier & Precision & Recall & $\begin{array}{c}\text { F1- } \\
\text { Measure }\end{array}$ & Accuracy & $\begin{array}{c}\text { ROC } \\
\text { Area }\end{array}$ \\
\hline \multicolumn{5}{|c|}{ Train+ (10-fold CV) } \\
\hline J48 & 0.990 & 0.990 & 0.990 & 99.04 & 0.991 \\
\hline RF & 0.990 & 0.990 & 0.990 & 99.04 & 0.997 \\
\hline LMT & 0.992 & 0.992 & 0.992 & 99.21 & 0.999 \\
\hline \multicolumn{5}{|c|}{ LC-NAF Test } \\
\hline J48 & 0.992 & 0.992 & 0.992 & 99.18 & 0.992 \\
\hline RF & 0.993 & 0.993 & 0.993 & 99.28 & 0.998 \\
\hline LMT & 0.993 & 0.993 & 0.993 & 99.31 & 0.999 \\
\hline \multicolumn{7}{|c|}{ UNT DL Test } \\
\hline J48 & 0.985 & 0.985 & 0.985 & 98.54 & 0.985 \\
\hline RF & 0.989 & 0.989 & 0.989 & 98.87 & 0.987 \\
\hline LMT & 0.989 & 0.989 & 0.989 & 98.91 & 0.991 \\
\hline
\end{tabular}

Table 1. Best performing classifiers on Train+ and test datasets

\section{REFERENCES}

Dublin Core Metadata Initiative. (2012). Dublin Core Metadata Element Set, Version 1.1. Retrieved from http://dublincore.org/documents/dces/

Heng, J., \& Grishman, R. (2006). Analysis and repair of name tagger errors. Proceedings of joint conference of the International Committee on Computational Linguistics and the Association for Computational Linguistics 420-427.

IBM (n.d). NameSiftr. InfoSphere Global Name Management 5.0.0. Retrieved from https://www.ibm.com/support/knowledgecenter/SSEV5M_5.0.0/com.ibm.iis.gnm.api.doc/topics/gnr_ns_api_intro-description.html

Library of Congress (2012). Dublin Core Metadata Element set mapping to MODS version 3. Retrieved from http://www.loc.gov/standards/mods/dcsimple-mods.html

Library of Congress (n.d). LC linked data service. Retrieved from http://id.loc.gov/download/

Library of Congress. Network Development and MARC Standards Office. (2016). MARC 21 format for bibliographic data. Retrieved from http://www.loc.gov/marc/bibliographic/

Mateos, P. 2007. A review of name-based ethnicity classification methods and their potential in population studies. Population Space and Place.

Paik, W., Liddy, E. D., Yu, E., \& McKenna, M. (1996). Categorizing and standardizing proper nouns for information retrieval. Corpus Processing for Lexical Acquisition p. 61-73

Schema.org (n.d.). Full hierarchy. http://schema.org/docs/full.htm 